Article

\title{
State-Population Narrowing Effect in Two-Photon Absorption for Intense Hard X-ray Pulses
}

\author{
Krzysztof Tyrała ${ }^{1}$, Klaudia Wojtaszek ${ }^{1}$, Marek Pajek ${ }^{1}$, Yves Kayser ${ }^{2}$, Christopher Milne ${ }^{3}$, \\ Jacinto Sá ${ }^{4,5}$ and Jakub Szlachetko ${ }^{1,4, *}$ \\ 1 Institute of Physics, Jan Kochanowski University, Kielce 25-001, Poland; krzysztof.tyrala1@wp.pl (K.T.); \\ klaudia.wojtaszek@interia.pl (K.W.); m.pajek@ujk.edu.pl (M.P.) \\ 2 Physikalisch-Technische Bundesanstalt (PTB), Berlin 10587, Germany; yves.kayser@ptb.de \\ 3 Paul Scherrer Institute, Villigen 5232, Switzerland; chris.milne@psi.ch \\ 4 Institute of Physical Chemistry, Polish Academy of Sciences, Warsaw 01-224, Poland; jacinto.sa@kemi.uu.se \\ 5 Department of Chemistry-Ånsgtröm Laboratory, Uppsala University, Uppsala 752 36, Sweden \\ * Correspondence: jakub.szlachetko@ujk.edu.pl; Tel.: +48-41-349-6440
}

Academic Editor: Kiyoshi Ueda

Received: 29 April 2017; Accepted: 21 June 2017; Published: 24 June 2017

\begin{abstract}
We report on studies of state-populations during the two-photon absorption process using intense $\mathrm{X}$-ray pulses. The calculations were performed in a time-dependent manner using a simple three-level model expressed by coupled rate equations. We show that the proposed approach describes well the measured rates of X-rays excited in the one-photon and two-photon absorption processes, and allows detailed investigation of the state population dynamics during the course of the incident X-ray pulse. Finally, we demonstrate that the nonlinear interaction of X-ray pulses with atoms leads to a time-narrowing of state populations. This narrowing-effect is attributed to a quadratic incidence X-ray intensity dependence characteristic for nonlinear interactions of photons with matter.
\end{abstract}

Keywords: X-ray nonlinear processes; two-photon absorption; rate equations

\section{Introduction}

In X-ray spectroscopy, the photon absorption and emission processes are usually treated within a weak photon-matter interaction limit, which implies that photon-in and photon-out processes are linearly correlated. However, for strong enough X-ray fields, the nonlinear regime may be accessed and multi-photon processes are possible, leading to a nonlinear dependence of the $\mathrm{X}$-ray photons in/out signals. Such a nonlinear regime, available for many years at optical frequencies in laser spectroscopy [1-4], remained an unexplored area at X-ray wavelengths due to the lack of strong enough X-ray sources. The ability to access nonlinear light-matter interactions at X-ray wavelengths became possible only recently thanks to the development of X-ray Free Electron Lasers (XFELs) $[5,6]$. In contrast to the optical laser wavelengths, the photon-atom interaction at hard X-ray wavelengths involves bound core-electrons, and leads to the excitation of intermediate electronic states with sub-femtosecond lifetimes [7-10]. Consequently, at femtosecond-durations, the intense pulses made available by XFELs presently allow us to access a thus-far-uninvestigated area of physics, and to probe the physical mechanisms that drive the nonlinear interaction of X-rays with matter [11-13].

With regard to applications, the two-photon absorption (TPA) process using hard X-rays allows access to different excitation states of matter, thanks to the specific selection rules for electronic transitions. In contrast to the one photon absorption (OPA) process, which is determined by dipole-allowed transitions, the TPA process requests a change in the electron orbital quantum number by \pm 2 or 0 , thus allowing access to quadrupole or forbidden excitations. This opens new avenues for 
studying unexplored electronic states of matter via two-photon absorption. However, the application of TPA at XFELs for studying electronic excitations is still problematic in practice. Because of low cross-sections, the TPA process is competing with other first- and second-order photon-atom interactions, which have to be considered, in particular, for ultrashort X-ray pulses. Consequently, knowledge of the time-evolution of the population of states, as well as on the subsequent X-ray emission process for ultrashort $\mathrm{X}$-ray pulses, is crucial in order to interpret the experimental results from XFELs.

In the present paper, we combine the results of a TPA/OPA experiment performed in the Linac Coherent Light Source (LCLS) [13], in which the X-rays indicating the TPA and OPA processes were measured, with time-dependent calculations allowing us to interpret the one- and two-photon absorption process for ultrashort hard X-ray pulses. In these calculations, we used a three-level atomic state model to describe the time-evolution of a population of excited electronic states during the course of a femtosecond-duration X-ray pulse. In this way, we could follow the time dependences of population states and subsequent $\mathrm{X}$-ray emission for both linear and nonlinear atomic response. The predictions of the presented model are in good agreement with the measured X-ray rates corresponding to one- and two-photon absorption in copper near the K-absorption edge. We also demonstrate a narrowing effect in population states generated through the nonlinear two-photon absorption mechanism.

\section{Materials and Methods}

For the calculations of two-photon absorption rates and time dependent population states, a three-level model described by rate equations was assumed. A similar solution was proposed in [13], where a constant intensity for the incident X-rays was assumed and, furthermore, a stationary case was considered. This allowed for an analytical solution of the rates equations. Herein, in contrast, we focus on an exact time-dependent solution of these rate equations for a given initially assumed time-profile of the incident X-ray pulse. Consequently, this approach allows the study of the time evolution of both the state population and the X-ray emission for a given pulse shape, i.e., a full description of the dynamics of the OPA and TPA processes. The schematic representation of atomic states included in the three-level rate equations model is shown in Figure 1.

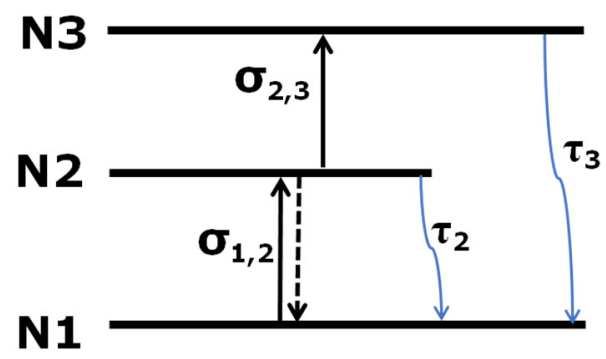

Figure 1. A schematic representation of the three-level model, representing the possible electronic transitions in one- and two-photon absorption processes on atomic core states. $\mathrm{N}_{1}$ corresponds to the population of the ground state, $\mathrm{N}_{2}$ to the population of the virtual intermediate state, and $\mathrm{N}_{3}$ to the ionized state. $\sigma_{1,2}$, and $\sigma_{1,2}$ are cross-sections for the first and second photon absorption. $\tau_{2}$ and $\tau_{3}$ corresponds to the lifetimes of the $\mathrm{N}_{2}$ and $\mathrm{N}_{3}$ states.

This model is based on the conclusions drawn from the experimental report [13], where the two-photon absorption mechanism leading to sequential ionization through an intermediate virtual atomic state was observed. In this 3-level model (see Figure 1), $\mathrm{N}_{1}$ corresponds to the population of the ground state, $\mathrm{N}_{2}$ to the population of the virtual intermediate state, and $\mathrm{N}_{3}$ to the ionized atom through the sequential $(1 \rightarrow 2 \rightarrow 3)$ two-photon absorption process. According to this picture, a subsequent radiative decay of the $\mathrm{N}_{2}$ state carries information on the direct OPA mechanism and a 
radiative decay of the $\mathrm{N}_{3}$ state on the TPA process. We assumed that the excitation of the $\mathrm{N}_{3}$ state is possible only through the intermediate $\mathrm{N}_{2}$ state, i.e., only a sequential two-photon absorption process is possible. Direct, simultaneous two-photon absorption is neglected here, because it is expected that the cross-sections for this process are three orders of magnitude lower than the sequential TPA mechanism [9].

Assuming a sequential model for the two-photon absorption mechanism, the following rate equations are considered:

$$
\begin{gathered}
\frac{\mathrm{dN}_{1}}{\mathrm{dt}}=-\mathrm{I}(\mathrm{t}) \times\left(\mathrm{N}_{1}(\mathrm{t}) \times \sigma_{1,2}-\mathrm{N}_{2}(\mathrm{t}) \times \sigma_{1,2}\right)+\frac{\mathrm{N}_{2}(t)}{\tau_{2}}+\frac{\mathrm{N}_{3}(t)}{\tau_{3}}, \\
\frac{\mathrm{dN} \mathrm{N}_{2}}{\mathrm{dt}}=\mathrm{I}(\mathrm{t}) \times\left(\mathrm{N}_{1}(\mathrm{t}) \times \sigma_{1,2}-\mathrm{N}_{2}(\mathrm{t}) \times \sigma_{2,3}-\mathrm{N}_{2}(\mathrm{t}) \times \sigma_{1,2}\right)-\frac{\mathrm{N}_{2}(t)}{\tau_{2}}, \\
\frac{\mathrm{dN_{3 }}}{\mathrm{dt}}=\mathrm{I}(\mathrm{t}) \times \mathrm{N}_{2}(\mathrm{t}) \times \sigma_{2,3}-\frac{\mathrm{N}_{3}(t)}{\tau_{3}}, \\
\mathrm{~N}_{1}(\mathrm{t})+\mathrm{N}_{2}(\mathrm{t})+\mathrm{N}_{3}(\mathrm{t})=\mathrm{N},
\end{gathered}
$$

where $I(t)$ describes the time structure of the incident X-ray pulse and $\mathrm{dN}_{1} / \mathrm{dt}, \mathrm{dN} \mathrm{N}_{2} / \mathrm{dt}, \mathrm{dN}_{3} / \mathrm{dt}$ are the decay rates of the initial, intermediate, and ionized states of an atom at time $t$. The cross-sections $\sigma_{1,2}$ and $\sigma_{2,3}$ represent transition probabilities between the $N_{1}$ and $N_{2}$, and the $N_{2}$ and $N_{3}$ states, respectively. In Equation (1), the first term describes the transitions between states $N_{1}$ and $N_{2}$ due to photon absorption and stimulated emission processes, respectively. The $N_{2} / \tau_{2}$ and $N_{3} / \tau_{3}$ terms in this equation describe the $\mathrm{N}_{1}$-state decay rates for radiative transitions from the $\mathrm{N}_{2}$ and $\mathrm{N}_{3}$ levels with lifetimes $\tau_{2}$ and $\tau_{3}$, respectively. Similarly, Equation (2) describes the $\mathrm{N}_{2}$ state which can be populated by photon absorption from the $\mathrm{N}_{1}$ level or alternatively depopulated either by absorption of a second photon moving the system to the $\mathrm{N}_{3}$ state or by stimulated emission as well as by adiative decay with lifetime $\tau_{2}$ to the $\mathrm{N}_{1}$-state. Finally, the $\mathrm{N}_{3}$ states are populated from the $\mathrm{N}_{2}$ state through the absorption of a second X-ray photon, and depopulated by a decay onto the $\mathrm{N}_{1}$ ground state with radiative lifetime $\tau_{3}$. The number of electrons $\mathrm{N}$ in the 3-level system is conserved, and the initial condition $\mathrm{N}_{1}(t=0)=\mathrm{N}$ and $\mathrm{N}_{2}(t=0)=\mathrm{N}_{3}(t=0)=0$ was assumed.

\section{Results}

\subsection{Details on the Computation}

In order to compare the predictions of the time-dependent $X$-ray emission rates obtained from the 3-level model with available experimental data, the calculations were performed for a copper atom and an incident $\mathrm{X}$-ray photon energy close to the $\mathrm{Cu}-\mathrm{K}$-shell edge. The details of the experimental setup and the data analysis can be found in [13]. Thanks to a relatively small detuning of only $12 \mathrm{eV}$ with respect to the ionization threshold, both one- and two-photon absorption channels were probed in this experiment by measuring simultaneously the $\mathrm{X}$-rays from a radiative decay of the intermediate state excited through inelastic scattering of the incident photons, as well as the non-resonant $\mathrm{K} \alpha_{1,2}$ $\mathrm{X}$-rays resulting from the two-photon absorption process. The $\mathrm{X}$-ray signatures of the TPA and OPA processes were measured with a high-resolution von Hamos diffraction spectrometer. The experiment thus provided information on the flux and incident $X$-ray energy dependences for the TPA mechanism. In the present calculations, the photon absorption cross sections for the $\mathrm{N}_{1} \rightarrow \mathrm{N}_{2}$ and $\mathrm{N}_{2} \rightarrow \mathrm{N}_{3}$ excitations were taken from the reported experimental values [13], which were determined to be $\sigma_{1,2}=5.5 \times 10^{-22} \mathrm{~cm}^{2}$ and $\sigma_{2,3}=1.4 \times 10^{-16} \mathrm{~cm}^{2}$. Similarly, the atomic lifetimes for the $\mathrm{N}_{2}$ and $\mathrm{N}_{3}$ states were fixed to $\tau_{2}=5.4 \times 10^{-17} \mathrm{~s}$ and $\tau_{3}=4.42 \times 10^{-17} \mathrm{~s}$, respectively. 
In the calculations, we assumed a Gaussian-shaped X-ray pulse, which was inserted into Equations (1)-(3) in the following form:

$$
I(t)=\frac{I_{0}}{\sqrt{2 \pi} \sigma} \times \exp \left(-\frac{(t)^{2}}{2 \sigma^{2}}\right)
$$

In the above equation, $\mathrm{I}_{0}$ represent the $\mathrm{X}$-ray pulse intensity, and $\sigma$ the standard deviation of its time profile. In the computations, we assumed a Gaussian X-ray pulse full width at half maximum value of $30 \mathrm{fs}$ as reported in the experiment. This approximation was made since the experiment took advantage of the self-seeded mode of operation of the LCLS XFEL [6], which transforms the broad, spiky SASE (Self-amplified Spontaneous Emission) spectral structure and reduces it to a quasi-monochromatic spectrum. The resulting X-ray pulse in time will be similarly a single pulse with limited structure [14]. The numerical calculations for the time-dependent population of states were performed in the range of $\Delta t=100 \mathrm{fs}$ (i.e., about 3 times the pulse length) and with a time-step (dt) of $10^{-4} \mathrm{fs}$, i.e., a value being much smaller than the pulse width and the lifetimes of the probed atomic states.

\subsection{X-ray Rates for One- and Two-Photon Absorption}

Based on the rate equations for the 3-level model described in Section 2, we first performed calculations on the absolute $X$-ray rates for the OPA and TPA processes versus the incident $X$-ray flux. With these computations, we could validate the time-dependent approach by comparing the calculated $\mathrm{X}$-ray rates with the previously reported experimental data. The calculated $\mathrm{X}$-ray rates were obtained by integrating the differential decay rates $\mathrm{dN}_{2} / \tau_{2}$ (OPA) and $\mathrm{dN}_{3} / \tau_{2}$ (TPA) over the time lapse of the incident $\mathrm{X}$-ray pulse. The results, presented in Figure 2, show a good agreement with the reported experimental results [13].

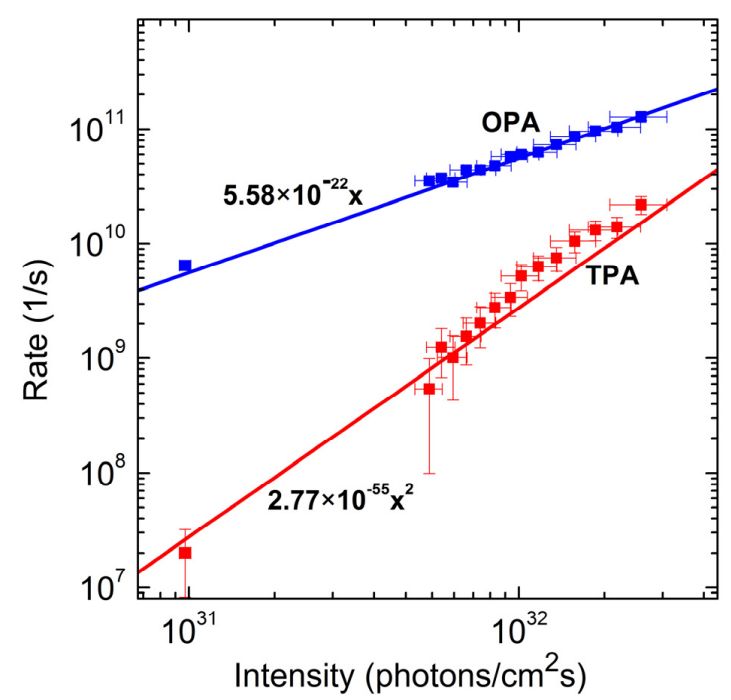

Figure 2. Comparison between calculated (solid line) and measured (squares) X-ray rates at different incidence X-ray flux for one photon absorption (OPA) (blue) and two-photon absorption (TPA) (red) processes. The experimental results were obtained at the Linac Coherent Light Source(LCLS) X-ray Free Electron Laser and are taken from Ref. [13].

In Figure 2, the measured X-ray emission rates corresponding to the OPA and TPA processes are plotted versus the fluence of the incident $X$-ray photons. The expected linear $(f=a x)$ and quadratic dependencies $\left(f=a x^{2}\right)$ of the measured rates for OPA and TPA processes, respectively, can be clearly seen. The calculated slope of the linear dependence of the OPA signal provides a one-photon absorption 
cross-section of $5.6 \times 10^{-22} \mathrm{~cm}^{2}$. Since the data are plotted on Log-Log scale, the TPA quadratic nature is distinguished from different curve slope as compared to OPA dependence. This value is in agreement with the reported experimental cross-section of $5.5 \times 10^{-22} \mathrm{~cm}^{2}$. The calculated coefficient of the quadratic function describing the TPA intensity is equal to $2.8 \times 10^{-55} \mathrm{~cm}^{4} \mathrm{~s}$, hence a qualitative agreement with the fitted experimental value of $4.1 \times 10^{-55} \mathrm{~cm}^{4} \mathrm{~s}$ is given. The obtained results validate, the assumptions made in the theoretical three-level system model, including assumption of a Gaussian shape for the temporal profile of the incident $X$-ray pulse.

\subsection{Time-Dependent Calculations for State Population}

The time-dependent approach employing the equations described in Section 2 allows for precise studies of the time evolution of populations in the ground, intermediate and final states during the course of an X-ray pulse. Figure 3 shows the time profiles of the population of the discussed states calculated for an incident X-ray flux of $1.5 \times 10^{32}$ photons $/ \mathrm{cm}^{2} \cdot \mathrm{s}$, which was a typical value used in the experiment discussed in Figure 2. For comparison, the shape of the incident $X$-ray pulse is also shown in Figure 3.

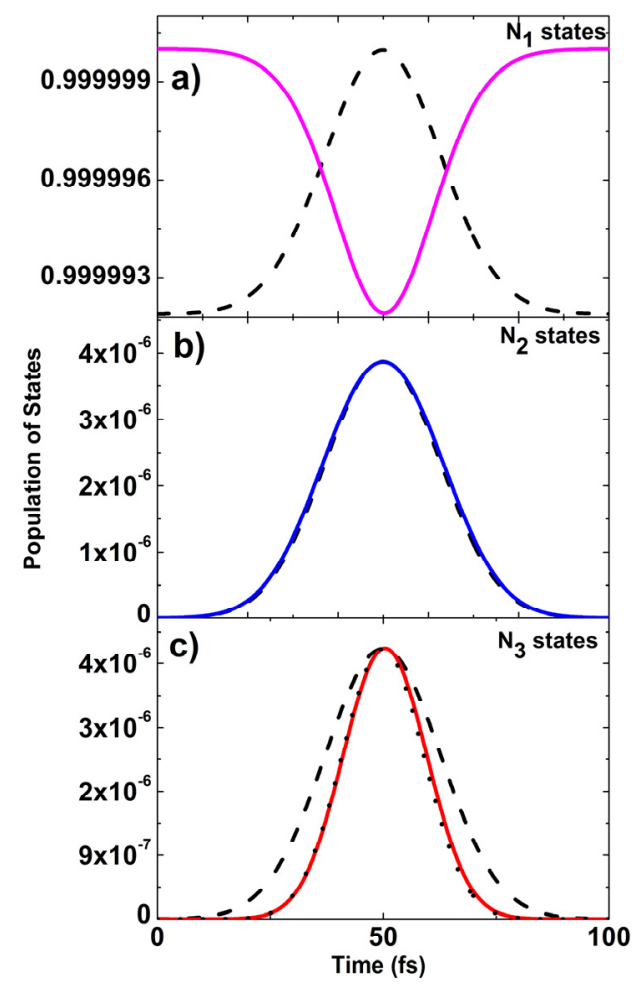

Figure 3. Calculated time-dependent population of the $\mathrm{N}_{1}, \mathrm{~N}_{2}$ and $\mathrm{N}_{3}$ states. The dashed line represents the shape of the incidence $X$-ray pulse, and is plotted in $(\mathbf{a}-\mathbf{c})$ for comparison. In Figure $\mathbf{c}$, we also plot the Gauss-square function (for details see text).

In Figure $3 a$, we show the population $\mathrm{N}_{1}$ of the ground state versus the time, which is anticorrelated with the time evolution of the incident X-ray pulse. Consequently, both curves exhibit the same width and extrema positions. After the incident X-ray pulse ends, the population of the ground state completely recovers its initial value $\mathrm{N}_{1}=\mathrm{N}$ through decays from the $\mathrm{N}_{2}$ and $\mathrm{N}_{3}$ states. The population of the intermediate state, plotted in Figure $3 b$, is fully correlated with the incident X-ray pulse and anticorrelated with the ground state. The biggest difference, shown in Figure 3c, is observed for a continuum state, which is populated by the TPA process. While the peak value in time for the $\mathrm{N}_{3}$ population state is at the same position as for the peak of the incident $X$-ray pulse, the width of the $\mathrm{N}_{3}$ population state evolution is significantly narrower. We find that, while the incident $\mathrm{X}$-ray 
pulse is $30 \mathrm{fs}$ long, the population of the $\mathrm{N}_{3}$ state can be characterized with a Gaussian shape of about $21 \mathrm{fs}$ width. Finally, as noticed above for the $\mathrm{N}_{1}$ state population recovery at the end of X-ray pulse, the populations of both the $\mathrm{N}_{2}$ and $\mathrm{N}_{3}$ states vanish to zero through the decays of these states to the ground state.

Using the presented 3-level model approach, we extracted the full widths at half maximum for the populations of the intermediate $\left(\mathrm{N}_{2}\right)$ and continuum $\left(\mathrm{N}_{3}\right)$ states for $X$-ray fluxes varying in the range of $10^{30}-10^{33}$ photons $/ \mathrm{cm}^{2} \mathrm{~s}$. The results are presented in Figure 4, which confirms our previous observations. The calculated population $\mathrm{N}_{3}$ of the continuum state presents a narrowing effect, which leads to a shorter time distribution of the states populated through the two-photon absorption. The performed calculations demonstrate that at higher X-ray flux, i.e., above $10^{32}$ photons $/ \mathrm{cm}^{2} \mathrm{~s}$, the widths of the populations of the intermediate and continuum states systematically increase (Figure 4). However, it should be noted that the difference between the widths of these states amounts to about $30 \%$ and is constant over the studied incidence $\mathrm{X}$-ray flux range.

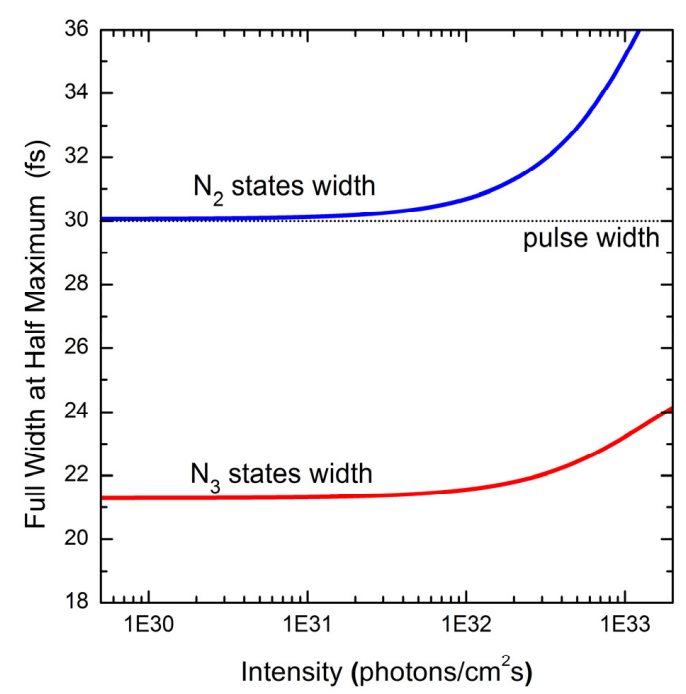

Figure 4. Calculated width (FWHM-full width at half maximum) for the $\mathrm{N}_{2}$ (blue) and $\mathrm{N}_{3}$ (red) states population as a function of the incident $X$-ray flux. The assumed width of the $X$-ray pulse was $30 \mathrm{fs}$ and is drawn for comparison as a horizontal dotted line.

\section{Discussion}

The presented calculations regarding the time evolution of the state populations and X-ray emission rates for the OPA and TPA processes can be discussed in different aspects. First, we would like to stress that the presented 3-level model is very simple in terms of number of atomic states, and hence the possible excitations and transitions, that are considered. We are aware that the XFEL beam interaction may lead, in specific cases, to more complicated distributions of atomic states that very often may be difficult or even impossible to treat with such a simple model. Nonetheless, as shown in the present report, the three-level model rate equations can be employed as a general tool for the description of nonlinear phenomena relevant for two-photon absorption via X-ray interaction with core atomic states.

The presented model describes quite well the reported experimental dependences of the X-ray emission rates on the incident $\mathrm{X}$-ray flux for both one- and two-photon absorption processes. The linear and quadratic dependences observed for the OPA and TPA processes were able to be well reproduced by the 3-level model calculations, and the extracted total-cross section values are in agreement with the experiment. The observed underestimation of the TPA cross-section by our calculations will be discussed below, together with the narrowing effect found for the population of the continuum state through the TPA mechanism. 
As shown in Figures 3 and 4, the population $\mathrm{N}_{3}$ of continuum states is about $30 \%$ narrower than the population $\mathrm{N}_{2}$ of the intermediate state and thus also narrower than the applied incident X-ray flux. While the incident X-ray flux width is $30 \mathrm{fs}$, the estimated width for the population of the continuum state is about $21.4 \mathrm{fs}$. We found that this effect can be explained by an effective square-dependence of the excitation of these atomic states on the incident photon flux due to the two-photon absorption process. In fact, this dependence manifests, in the occurrence of the nonlinear $\mathrm{X}$-ray interaction with matter. Indeed, the two-photon absorption process scales with the square of the applied X-ray intensity. Therefore, the employed Gaussian-like incident X-ray pulse will effectively act as the squared Gaussian profile. The Gauss-square function, in comparison with the nominal Gauss-shape function, will exhibit a width that is narrower by a factor of $2^{-1 / 2}$ (i.e., $\approx 1.41$ ). Indeed, this observation is in perfect agreement with the observed narrowing, i.e., the ratio of $30 \mathrm{fs}$ to $21.4 \mathrm{fs}$ is equal to 1.4. This is also confirmed in Figure 3c, where the Gaussian-square function (dotted line) is compared with the calculated population of the continuum state, and both profiles are in perfect agreement. Finally, we would like to note that the discussed narrowing effect should be taken into consideration, in general, for the cross-section determination of nonlinear processes. As reported in [13], the experimental data were normalized while assuming that the incident X-ray pulse has a Gaussian shape. While this normalization is valid for linear processes, it seems that in nonlinear interaction cases the normalization factor resulting from the time distribution of the incident X-ray flux should be, in addition, divided by a factor if $2^{-1 / 2}$ for the two-photon processes. It is expected that, for this reason, the cross-sections calculated here for TPA mechanism should again be lower, by a factor of about 1.4, than the discussed experimental results.

Finally, we would like to comment on the observed increase of widths of population profiles for excited states, here both $\mathrm{N}_{2}$ and $\mathrm{N}_{3}$, at higher incidence X-ray flux. As can be seen in Figure 4, for incident X-ray fluxes above $10^{32}$ photons $/ \mathrm{cm}^{2} \mathrm{~s}$, both time profiles for these states become wider, however the ratio of their widths remains constant and is equal to about 1.4. The observed increase in the width for the OPA and TPA processes may be considered as a first indication that the saturation regime for nonlinear interaction is approached $[15,16]$. The atomic state response and state populations no longer follow the incident photon pulse profile, and therefore cannot be evaluated with a simple Gaussian-like approximation. Indeed, the saturation of absorption effects were reported for metallic Fe at X-ray flux intensities of $8.4 \times 10^{34}$ photons $/ \mathrm{cm}^{2} \cdot \mathrm{s}$ [16]. At the present point, the studies of such effects need more theoretical investigation, as well as experimental verifications.

Though the present simulations were performed with a simplified Gaussian-like pulse structure, this is still a good approximation for single-shot pulse shapes expected during self-seeded operation of an XFEL [6,14]. In addition, since the experimental data was taken in a cumulative measurement where many pulses were summed together to improve the signal-to-noise, a Gaussian pulse shape is a good representation of the average incident pulse shape. We show that the reported narrowing of atomic states populated through nonlinear interaction relates to a square-dependence of the applied $\mathrm{X}$-ray pulse intensity not to the incident $\mathrm{X}$-ray pulse shape. Further research should address other phenomena that may be related to the single-pulse interaction with SASE-like structure. For example, one may investigate the structure and envelope of the applied X-ray pulse. Moreover, different parameters describing the SASE structure of the incident beam may be evaluated, like spike width and spike separation in the time domain. We anticipate that these effects will start to become significant as they approach the timescales of atomic core-hole lifetimes. For any such single-shot simulations and experiments not only does the experiment require precise measurement of the incident beam properties, but the simulation parameters need to be carefully tuned to match the specific XFEL facility at which the experiment takes place.

Acknowledgments: J. Szlachetko acknowledges the National Science Centre (NCN), Poland for support under grant no. 2015/19/B/ST2/00931. J. Szacknowledges the Polish Ministry of Science and Higher Education for support from the budget for science in 2016-2019 under grant IDEAS Plus II IdPII 2015000164. 
Author Contributions: J.Sz. conceived and designed the project, M.P. delivered the rate equations and K.T. executed calculations and prepared the figures as well as the initial version of the manuscript. All authors participated in result discussions and manuscript preparation. J.Sz. wrote the manuscript with input from K.T., K.W., Y.K., M.P. C.M. and J.Sá.

Conflicts of Interest: The authors declare no conflict of interest.

\section{References}

1. Keller, U. Recent developments in compact ultrafast lasers. Nature 2003, 424, 831-838. [CrossRef] [PubMed]

2. Schuster, I.; Kubanek, A.; Fuhrmanek, A.; Puppe, T.; Pinkse, P.W.H.; Murr, K.; Rempe, G. Nonlinear spectroscopy of photons bound to one atom. Nat. Phys. 2008, 4, 382-385. [CrossRef]

3. Srinivasan, K.; Painter, O. Linear and nonlinear optical spectroscopy of a strongly coupled microdiskquantum dot system. Nature 2007, 450, 862-865. [CrossRef] [PubMed]

4. Hori, M.; Sótér, A.; Barna, D.; Dax, A.; Hayano, R.S.; Friedreich, S.; Juhász, B.; Pask, T.; Widmann, E.; Horváth, D.; et al. Two-photon laser spectroscopy of antiprotonic helium and the antiproton-to-electron mass ratio. Nature 2011, 475, 484-488. [CrossRef] [PubMed]

5. Emma, P.; Akre, R.; Arthur, J.; Bionta, R.; Bostedt, C.; Bozek, J.; Brachmann, A.; Bucksbaum, P.; Coffee, R.; Decker, F.-J.; et al. First lasing and operation of an ångstrom-wavelength free-electron laser. Nat. Photonics 2010, 4, 641-647. [CrossRef]

6. Amann, J.; Berg, W.; Blank, V.; Decker, F.-J.; Ding, Y.; Emma, P.; Feng, Y.; Frisch, J.; Fritz, D.; Hastings, J.; et al. Demonstration of self-seeding in a hard-X-ray free-electron laser. Nat. Photonics 2012, 6, 693-698. [CrossRef]

7. Young, L.; Kanter, E. P.; Krässig, B.; Li, Y.; March, A.M.; Pratt, S.T.; Santra, R.; Southworth, S.H.; Rohringer, N.; DiMauro, L.F.; et al. Femtosecond electronic response of atoms to ultra-intense X-rays. Nature 2010, 466, 56-61. [CrossRef] [PubMed]

8. Tamasaku, K.; Nagasono, M.; Iwayama, H.; Shigemasa, E.; Inubushi, Y.; Tanaka, T.; Tono, K.; Togashi, T.; Sato, T.; Katayama, T.; et al. Double Core-Hole Creation by Sequential Attosecond Photoionization. Phys. Rev. Lett. 2013, 111, 043001. [CrossRef] [PubMed]

9. Tamasaku, K.; Shigemasa, E.; Inubushi, Y.; Katayama, T. X-ray two-photon absorption competing against single and sequential multiphoton processes. Nature 2014, 8, 313-316. [CrossRef]

10. Rohringer, N.; Ryan, D.; London, R.A.; Purvis, M.; Albert, F.; Dunn, J.; Bozek, J.D.; Bostedt, C.; Graf, A.; Hill, R.; et al. Atomic inner-shell X-ray laser at 1.46 nanometres pumped by an X-ray free-electron laser. Nature 2012, 481, 488-491. [CrossRef] [PubMed]

11. Beye, M.; Schreck, S.; Sorgenfrei, F.; Trabant, C.; Pontius, N.; Schüßler-Langeheine, C.; Wurth, W.; Föhlisch, A. Stimulated X-ray emission for materials science. Nature 2013, 11. [CrossRef] [PubMed]

12. Vinko, S.M.; Ciricosta, O.; Cho, B.I.; Engelhorn, K.; Chung, H.-K.; Brown, C.R.D.; Burian, T.; Chalupský, J.; Falcone, R.W.; Graves, C.; et al. Creation and diagnosis of a solid-density plasma with an X-ray free-electron laser. Nature 2012, 482, 59-62. [CrossRef] [PubMed]

13. Szlachetko, J.; Nachtegaal, M.; De Boni, E.; Willimann, M.; Safonova, O.; Sa, J.; Smolentsev, G.; Szlachetko, M.; Van Bokhoven, J.A.; Dousse, J.C.; et al. Establishing nonlinearity thresholds with ultraintense X-ray pulses. Sci. Rep. 2016, 6, 33292. [CrossRef] [PubMed]

14. Geloni, G.; Kocharyan, V.; Saldin, E. A novel self-seeding scheme for hard X-ray FELs. J. Mod. Opt. 2011, 58, 1391-1403. [CrossRef]

15. Nagler, B.; Zastrau, U.; Fäustlin, R.R.; Vinko, S.M.; Whitcher, T.; Nelson, A.J.; Sobierajski, R.; Krzywinski, J.; Chalupsky, J.; Abreu, E.; et al. Turning solid aluminium transparent by intense soft X-ray photoionization. Nat. Phys. 2009, 5, 693-696. [CrossRef]

16. Yoneda, H.; Inubushi, Y.; Yabashi, M.; Katayama, T.; Ishikawa, T.; Ohashi, H.; Yumoto, H.; Yamauchi, K.; Mimura, H.; Kitamura, H. Saturable absorption of intense hard X-rays in iron. Nat. Commun. 2014, 5, 5080. [CrossRef] [PubMed]

(C) 2017 by the authors. Licensee MDPI, Basel, Switzerland. This article is an open access article distributed under the terms and conditions of the Creative Commons Attribution (CC BY) license (http:/ / creativecommons.org/licenses/by/4.0/). 